\title{
VIII.
}

Aus dem Institut für exper. Pharmakologie der Úniversität Lemberg. (Direktor: Prof. Dr. L. Popielski).

\section{Zur Wirkung intravenöser Einspritzungen von konzentrierten Salz- und Zuckerlösungen. \\ Von}

Dr. G. G. Wilenko.

Über die Funktionsveränderungen, die die Einführung von konzentrierten Salzlösungen ins Blut hervorruft, sind wir nur wenig unterrichtet, denn die meisten Arbeiten auf diesem Gebiete (Klikowicz, Münzer, Heidenheim, Hamburger, Moritz, Magnus (1) sind nur mit Bezug auf die osmotischen Verhältnisse unternommen worden. Aus dem bis nun Bekannten sei hervorgehoben: Steigerung der Diurese, Zunahme der Gerinnbarkeit des Blutes (v. d. Velden (2), Reizung des Nervensystems (Münzer (3), Veränderungen in der Sekretion des Magensaftes (Wilenko (4), and endlich die Herabsetzung des Adrenalingykosurie (Tomaszewski und Wilenko (5).

Die letzte Beobachtung hat auch den Anlaß gegeben zur Untersuchung, wie die Einführung von konzentrierten Salzlösungen den Kohlehydratstoffwechsel beeinfluist.

Zuerst wurden Versuche mit 20 proz. Na-Cl Lösung, die, erwärmt, langsam in die Ohrvene des Kaninchens eingespritzt wurde, ausgeführt.

Aus der Tabelle I ist zu ersehen, daß der Eingriff eine bede uten de Hyperglykämie hervorruft, die bei Kaninchen auch nach zweitägigem Hungern zum Vorschein tritt und die bis vier Stunden andauern kann. Die Intensität der Hyperglykämie schwankt zwischen 0.16 Proz. und 0.56 Proz, beträgt aber zumeist ca. 0.25 Proz. Es war dabei noch ein möglicher Fehler auszuschließen. Aus den Untersuchungen von Magnus (1) wissen wir, daß zwecks Herstellung normaler osmotischer Verbältnisse ein Austausch von Wasser- und Salz- 
Tabelle I (Kochsalzversuche).

Warme, 20proz. Kochsalzlösung wurde in die Ohrvene der Kaninchen injiziert. Der Harn wurde bis zum folgenden Tag gesammelt (wo nicht anders angegeben ist), mit Katheter begrenzt und der nächste Harn wiederum kontrolliert. Die Zuckerbestimmungen im Harn polarimetrisch, der Blutzucker nach Schenk oder Frank und Moekel. Die Zeit der Blutentnahme immer angegeben.

\begin{tabular}{|c|c|c|c|c|c|}
\hline $\begin{array}{c}\text { Versuchs- } \\
\text { nummer } \\
\text { Datum }\end{array}$ & $\begin{array}{l}\text { Gewicht } \\
\text { Nahrung }\end{array}$ & $\begin{array}{c}\text { Zeit, Menge und } \\
\text { Konzentration der } \\
\text { Injektion }\end{array}$ & Harn & Blut & $\begin{array}{l}\text { Bemer- } \\
\text { kungen }\end{array}$ \\
\hline $\begin{array}{c}\text { VII } \\
\text { 31.X.1910 }\end{array}$ & $\begin{array}{l}2700 \mathrm{~g} \\
\text { gemischte } \\
\text { Kost }\end{array}$ & $\begin{array}{c}1 \text { Uhr } 48-59 \\
10 \mathrm{ccm} 15 \text { proz. NaCl} \\
5 \text { Uhr } 30-34 \\
10 \mathrm{ccm} 15 \text { proz. } \mathrm{NaCl}\end{array}$ & \begin{tabular}{|} 
bis 4 Uhr 45 \\
40 ccm zucker- \\
freien Harns \\
bis 5 Uhr 55 \\
45 ccm zucker- \\
freien Harns \\
bis 7 Uhr 20 \\
20 cem zucker- \\
freien Harns
\end{tabular} & 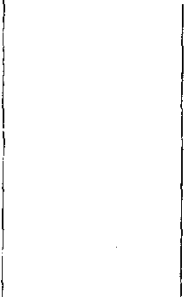 & \\
\hline $\begin{array}{l}\text { VIII } \\
\text { 2. XI. }\end{array}$ & $\begin{array}{l}2010 \mathrm{~g} \\
\text { Rüben }\end{array}$ & $\mid \begin{array}{c}1 \text { Uhr } 31-36 \\
7,5 \mathrm{ccm} 20 \text { proz. } \mathrm{NaCl} \\
6 \mathrm{Uhr} 11-15 \\
7,5 \mathrm{ccm} 20 \text { proz. } \mathrm{NaCl}\end{array}$ & $\begin{array}{c}125 \mathrm{ccm} \text { zu cker" } \\
\text { freien Harns }\end{array}$ & & \\
\hline $\begin{array}{l}\text { IX } \\
\text { 4. XI. }\end{array}$ & $\begin{array}{l}1350 \mathrm{~g} \\
\text { Rüben }\end{array}$ & $\begin{array}{c}12 \text { Uhr } 5-95 \mathrm{ccm} \\
20 \text { proz. } \mathrm{NaCl} \\
1 \text { Uhr } 8-124,5 \mathrm{ccm} \\
20 \text { proz. } \mathrm{NaCl} \\
2 \mathrm{Uhr} 8-125 \mathrm{ccm} \\
20 \text { proz. } \mathrm{NaCl}\end{array}$ & $\begin{array}{c}\text { bis } 2 \text { Uhr } 8 \\
85 \text { ccm Harn mit } \\
0,085 \mathrm{~g} \text { Zucker } \\
\text { bis } 0 \text { Uhr } \\
40 \mathrm{ccm} \text { zucker- } \\
\text { freien Harns }\end{array}$ & & \\
\hline $\begin{array}{c}\mathrm{X} \\
15 . \mathrm{XI} .\end{array}$ & $\begin{array}{l}1300 \mathrm{~g} \\
\text { Rüben }\end{array}$ & $\mid \begin{array}{c}7 \text { Uhr } 25-27 \\
5,5 \mathrm{ccm} 20 \text { proz. NaCl} \\
7 \text { Uhr } 49-51 \\
5 \text { cem } 20 \text { proz. NaCl }\end{array}$ & $\begin{array}{l}\text { bis } 7 \text { Uhr } 51 \text { din- } \\
\text { retischer, zucker- } \\
\text { freier Harn } \\
\text { von } 7 \text { Uhr } 51 \\
21 \text { ccm Harn mit } \\
0,05 \text { g Zacker } \\
\text { Eiwei } \beta \text { deutlich }\end{array}$ & $\left|\begin{array}{l}8 \text {. Uhr } 10 \text { ent- } \\
\text { nommen Blut } \\
0,16 \% \text { Zucker }\end{array}\right|$ & \\
\hline $\begin{array}{c}\mathrm{XI} \\
\text { 17. XI. }\end{array}$ & $\begin{array}{l}3200 \mathrm{~g} \\
\text { Rüben }\end{array}$ & $\mid \begin{array}{c}1 \mathrm{Uhr} 12-1511 \mathrm{ccm} \\
20 \text { proz. NaCl } \\
1 \mathrm{Uhr} 35-3910 \mathrm{ccm} \\
20 \text { proz. NaCl }\end{array}$ & $\begin{array}{c}\text { bis } 1 \text { Uhr } 35 \\
40 \text { ccm zucker- } \\
\text { freien Harns } \\
\text { bis } 2 \text { Uhr } 90 \mathrm{ccm} \\
\text { mit } 0,3 \mathrm{~g} \text { Zucker } \\
\text { bis } 5 \text { Uhr } 30 \\
24 \text { ccm mit } 0,5 \mathrm{~g} \\
\text { Zucker }\end{array}$ & $\begin{array}{l}1 \text { Uhr } 52 \text { Blut- } \\
\text { entnahme } \\
0,56 \% \text { Zucker }\end{array}$ & \\
\hline
\end{tabular}


Zur Wirkung intrav. Einspritzungen von konz. Salz- und Zuckerlösungen. 14j

Tabelle I (Fortsetzung).

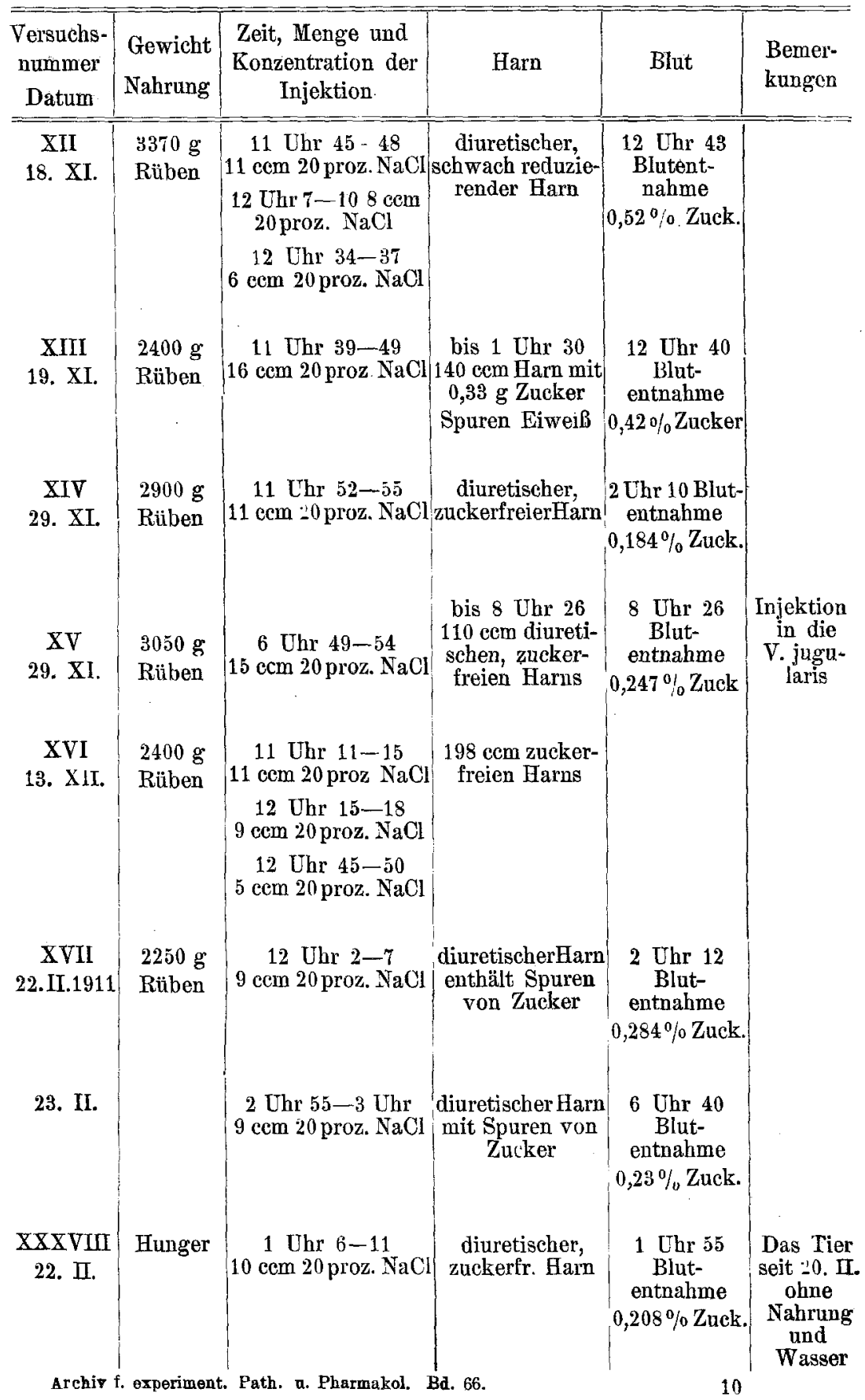


molekeln zwischen Blut und Geweben im Verlaufe von 100 Minuten stattfindet. Es war denkbar, daß die Blutentnahme in einem Zeitpunkte einer Wasserverarmung geschehen ist und infolgedessen ein höherer Blutzuckergehalt vorgetäuscht wurde. Die Tatsache der gesteigerten Diurese sprach schon dagegen, doch wurden direkte Bestimmungen der Trockensubstanz des Blutes ausgeführt.

Tabelle II.

Bestimmung der Trockensubstanz im Blute.

\begin{tabular}{|c|c|c|}
\hline $\begin{array}{c}\text { Tier } \\
\text { Datum }\end{array}$ & $\begin{array}{l}\text { Zeit } \\
\text { Uhr }\end{array}$ & \\
\hline $\mathrm{X}$ & 7 Chr 25 & $5,5 \mathrm{ccm} 20$ proz. NaCl-Lösung in die Ohrvene eingeführt \\
\hline 15. XI. & $\begin{array}{lll}7 & 49 \\
8 & \# & 10\end{array}$ & 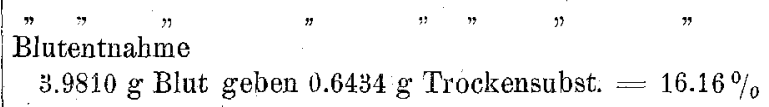 \\
\hline $\begin{array}{c}\mathrm{XI} \\
1 \mathrm{~T} . \mathrm{XI} .\end{array}$ & $\begin{array}{l}1 \text { Uhr } \\
1 \text { Uhu } 12\end{array}$ & $\begin{array}{l}\text { Blutentnahme } \\
4.2822 \mathrm{~g} \text { Blut geben } 0.7822 \mathrm{~g} \text { Trockensubst. }=18.32 \% \\
11 \mathrm{ccm} 20 \text { proz. NaCl in die Ohrvene eingeführt }\end{array}$ \\
\hline & $\begin{array}{lll}1 & y & 3 \check{5} \\
1 & \prime & 51\end{array}$ & 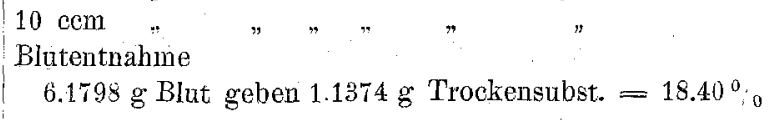 \\
\hline XII & 11 Uhr $4 \bar{\jmath}$ & $11 \mathrm{cem} 20$ proz. $\mathrm{NaCl}$ in die Ohrvene eingefühnt \\
\hline 18. XI. & $\begin{array}{lll}12 & \cdots & 7 \\
12 & \# & 34 \\
12 & \# & 42\end{array}$ & 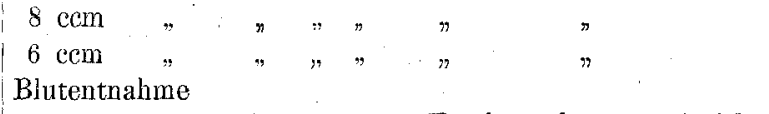 \\
\hline
\end{tabular}

Die Schwankungen des Wassergehaltes entsprechen den physiologischen Grenzen und können nicht zur Erklärung der Hyperglykämie herangezogen werden.

Aus der Tabelle I sehen wir weiter, daß der diuretische Harn nur wenig oder gar keinen Zucker enthält. Sogar bei sebr starker Hyperglykämie, über 0.5 Proz. sind im Harn nur Spuren von Zucker enthalten. Der Befund ist um so merkwürdiger, da nach üblicher Anschanung die Diurese den Übertritt des Blutzuckers in den Harn bedeutend erleichtern soll. (Pollak 6). In unseren Versuchen ist aber ein ganz entgegengesetztes Verhalten zu beobachten.

Die Tatsache ist nur dadurch zu erklären, daß die Niere für die Zuckermoleküle weniger durchgängig geworden ist. Diese Feststellung ist aber von weitgehender Bedeutung für unsere 
Vorstellungen über das Zustandekommen der Glykosurie, denn sie zeigt aufs dentlichste, daß das Verhalten der Niere dem Blutzucker gegenüber nicht als rein passiv den physikalischen Filtrationsgesetzen entspreehend zu betrachten ist; daß vielmebr die Niere unabhängig von der Konzentration des Zuckers im Blute den Übertritt der Glykose in den Harn selbständig zu regulieren imstande ist. Dieser Umstand muß fortan sowohl bei den experimentellen Glykosurien, wie auch beim menschlichen Diabetes berücksichtigt werden. Der Harn gibt infolge der selbständigen Nierentätigkeit nicht immer die Verhältnisse im Blute richtig wieder.

Die histologische Untersuchung ${ }^{1}$ ) von Herm Dozenten Nowicki hat eine Hyperämie in den Bowmanischen Kapseln und leichte Quellung der Epithelien der Tubuli contorti ergeben.

Wir sind dann der Frage näher getreten, wo der Ursprung der beobachteten Hyperglykämie zu suchen ist und zu welcher Gruppe der schon bekannten, sie einzureihen wäre. Zu diesem Zwecke haben wir die Salzlösung Kaninchen mit durchsebnittenen nervi splanchnici eingespritzt. Die Durebschneidung geschah nach Schulze (7).

Die Tabelle III zeigt, daß bei diesen Tieren die Hyperglykämie ausgeblieben ist. Die intravenöse Einführung von konzentrierter Salzlösung wirkt also ähnlich dem Piqûre von Claude Bernard, das heibt, reizt das zentrale Nervensystem.

Um einen näheren Einblick in die weiteren Mechanismen der Hyperglykämie za gewinnen, haben wir Glykogenbestimmungen in Leber und Muskeln ausgeführt, nachdem den Kaninchen dreimal die Salzlösung ins Blut gespritzt wurde. (Siehe Tab. IV.)

Wir sehen, daß die Muskeln fast ihr ganzes Glykogen abgegeben haben ( 0.006 bis 0.013 Proz. gegenüber dem normalen Gehalt von 0.4 Proz.); der Glykogengehalt der Leber liegt noch in den physiologischen Grenzen; doch ist es nicht ausgeschlossen, dab auch diese einen Teil ihres Glykogens eingebüßt hat, nur ist der Nachweis des Verlustes angesichts des Reichtums und der Schwankungen des Glykogens in der Norm, schwer festzustellen.

Es hat sich dann die Frage erhoben, wodurch eigentlich die konzentrierte Salzlösung wirksam ist; durch ihr wasserentziehendes

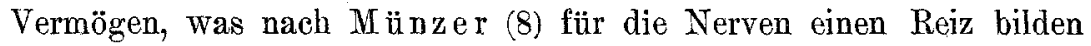
kann, oder durch ihre Joneneigenschaften.

1) Ich will auch an dieser Stelle Herrn Dozenten Nowichi dafür meinen herzlichsten Dank aussprechen. 


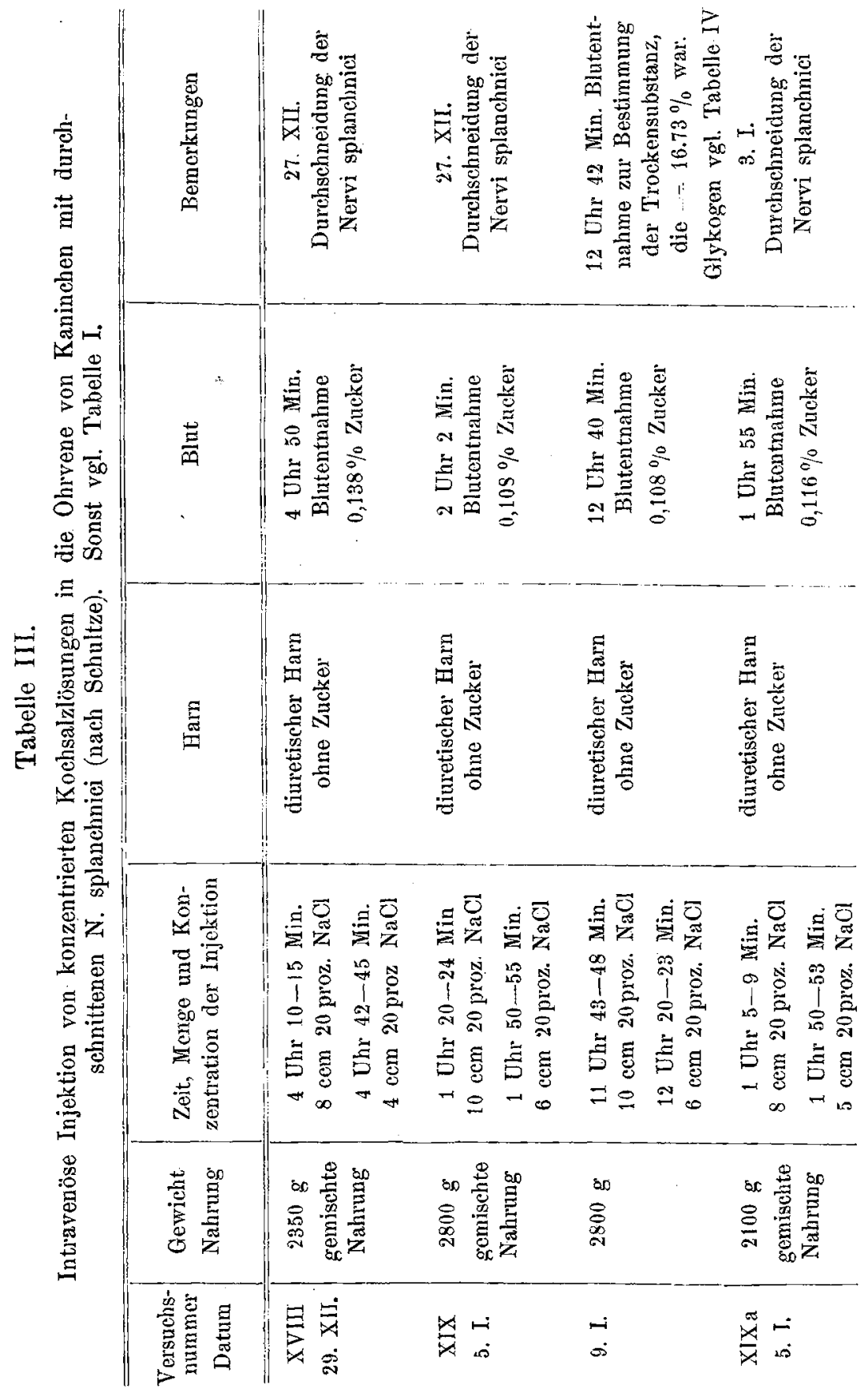


Zur Wirkung intrav. Einspritaungen von konz. Salz- und Zuckerlösungen. 149

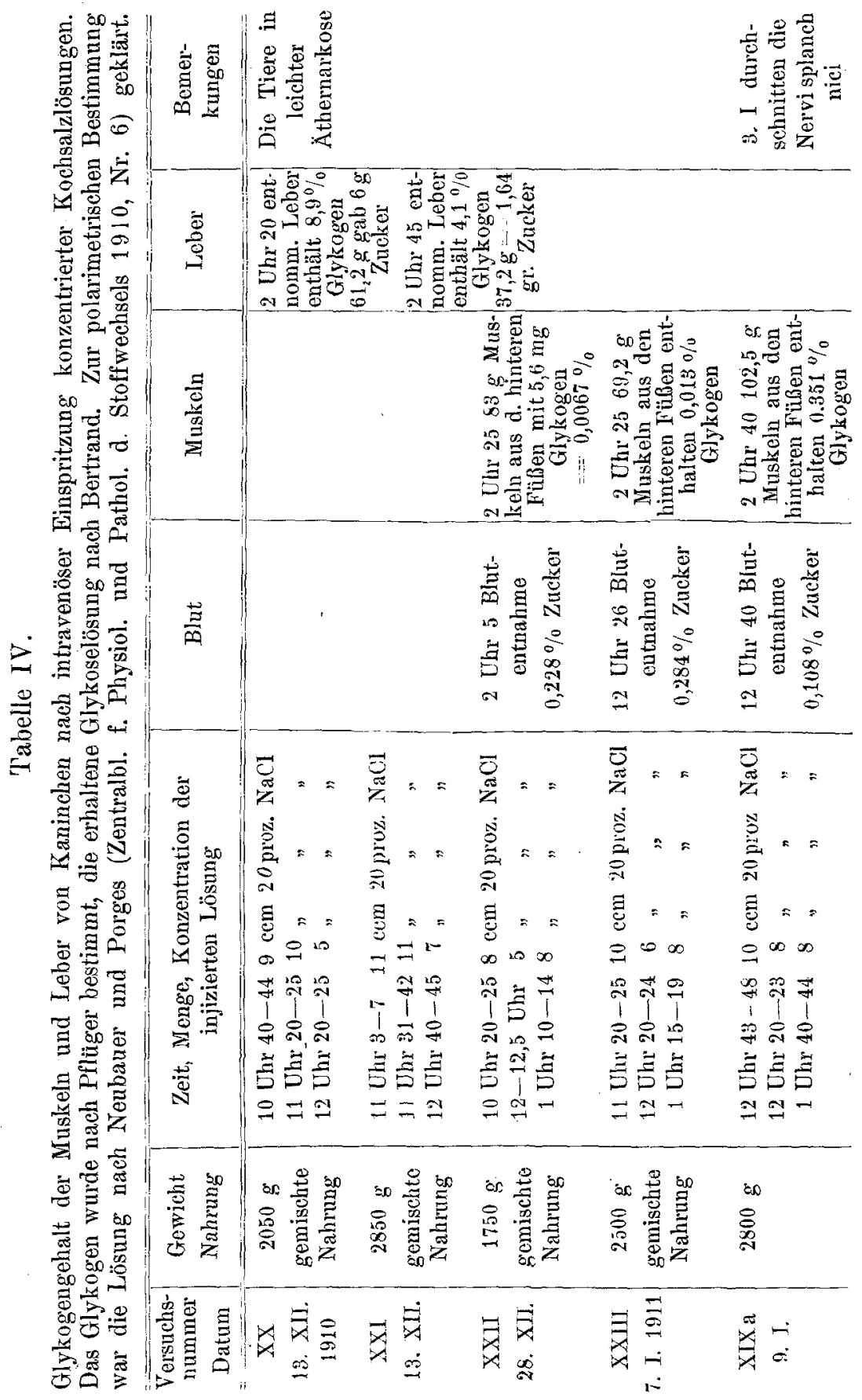


Die Anionfunktion haben wir dadurch geprüft, daß wir anstatt der NaCl-Lösung, eine annähernd äquimolekulare $\mathrm{Na}_{2} \mathrm{SO}_{4}$-Lösung in die Vene eingespritzt haben.

Die Tab. V zeigt, dal dem Anion beim Zustandekommen der Hyperglykämie keine besondere Bedeutung zuzuschreiben ist. Sowohl $\mathrm{NaCl}$, wie $\mathrm{Na}_{2} \mathrm{SO}_{4}$ rufen Hyperglykämie hervor. Auch der Niere gegenüber ist kein Unterschied zu bemerken. Es fällt sogar auf, đaß das $\mathrm{Na}_{2} \mathrm{SO}_{4}$ trotz seiner gröberen, im Vergleich zu NaCl, diuretischen Wirkung - die Glykosurie anscheinend noch stärker hemmt. A uch die Nierendu rchlässigkeit fü $r$ Zucker ist von Anion unabhängig.

Um die Bedeutung des Kations für die Hyperglykämie näher kennen zu lernen, wurde zur $\mathrm{NaCl}$-Lösung $\mathrm{KCl}$ und $\mathrm{CaCl}_{2}$ zugesetzt bei fast gleichbleibender molekularer Konzentration.

Aus den angeführten Zahlen (Tab. VI) ist zu ersehen, dab es unter diesen Umständen nicht zur Hyperglykämie kommt. Auch die Muskeln weisen einen normalen Glykogengehalt auf. (Tab. IV). Die hyperglykämische Wirkung der konzentrierten NaCl-Lösung berubt also auf einer Störung des pbysiologischen Verhältnisses unter den einzelnen Kationen, was einen Reiz fürs zentrale Nervensystem bildet. Ähnliche Tatsachen sind schon aus den Versuchen ron Overton, Höber und Loeb (9) bekannt und liefern einen nenerlichen Beweis, daß auch die Salze zu denjenigen Faktoren gehören, die die Protoplasmafunktion bestimmen.

Das hier nicht die Wertigkeit der Jonen ausschlaggebend ist, ist aus den Versuchen mit Zusatz von nur $\mathrm{CaCl}_{2}$ zu ersehen. Das Zweiwertige Ca kann nicht die NaCl-Hyperglykämie verhindern. Wir erinnern auch an die von Stoklasa festgestellte Bedeutung des $\mathrm{K}$ für die Oxydationsprozesse in der Pflanze ${ }^{1}$ ).

Um die Frage des Kations bei der Wirkung der konzentrierten NaCl-Lösung auf die Nierendurchlässigkeit für Zucker zu entscheiden konnte nicht derselbe Weg eingeschlagen werden, denn die Kationenmischung ruft keine Hyperglykämie hervor.

Es mußte also für das Kation in anderer Weise vorgegangen werden.

1) Bei der von Bock und Hoffmann (10) entdeckten, danin von Brown (11) und Fischer (12) näher studierten Glykosurie nach intravenöser Einführung von verdïnnten Kochsalzlösungen dürfte es sich um ähnliche Mechanismen handeln. Allerdings fehlen die Bestimmungen des Blutzuckers. 
Zur Wirkung intrav. Einspritzangen von konz. Salz- und Zackerlösungen. 151

Tabelle V.

Intravenöse Injektion konzentrierter Lösungen von $\mathrm{Na}_{2} \mathrm{SO}_{4}$.

\begin{tabular}{|c|c|c|c|c|c|}
\hline $\begin{array}{c}\text { Versuchs- } \\
\text { Nummer } \\
\text { Datum }\end{array}$ & $\begin{array}{l}\text { Gowicht } \\
\text { Nahrung }\end{array}$ & $\begin{array}{l}\text { Zeit, Menge, } \\
\text { Konzentration } \\
\text { der Injektion }\end{array}$ & Harn & Blut & $\begin{array}{l}\text { Bemer- } \\
\text { kungen }\end{array}$ \\
\hline $\begin{array}{l}\text { XXIV. } \\
1 \text { XII. } \\
1910\end{array}$ & $\begin{array}{c}3000 \mathrm{~g} \\
\text { gemischte } \\
\text { Nahrung }\end{array}$ & $\begin{array}{c}6 \mathrm{Uhr}-6 \mathrm{Uhr} 6 \\
17,5 \mathrm{ccm} \\
25 \% \mathrm{Na}_{2} \mathrm{SO}_{4} \\
\text { (Na2 } \mathrm{SO}_{4} \text { wasser- } \\
\text { frei berechnet) }\end{array}$ & $\begin{array}{l}\text { Diuretischer } \\
\text { Harn zuckerfrei } \\
\text { Spuren Eiweiß }\end{array}$ & $\begin{array}{c}6 \text { Uhr } 35 \\
\text { Blutentnahme } \\
0,206 \% \\
\text { Zueker }\end{array}$ & \\
\hline $\begin{array}{l}\text { XXV. } \\
\text { 1. XII. }\end{array}$ & $\begin{array}{c}1500 \mathrm{~g} \\
\text { gemischte } \\
\text { Nahrung }\end{array}$ & $\begin{array}{l}6 \mathrm{Uhr} 35-40 \\
9 \mathrm{ccm} \\
25 \% \mathrm{Na}_{2} \mathrm{SO}_{4}\end{array}$ & $\begin{array}{l}\text { Diuretischer } \\
\text { Harn zucker- } \\
\text { und eiweibfrei }\end{array}$ & $\begin{array}{c}7 \text { Uhr } 6 \\
\text { Blutentnahme } \\
0,30 \% \\
\text { Zucker }\end{array}$ & \\
\hline $\begin{array}{l}\text { XXVI. } \\
\text { 9. III. 1911 }\end{array}$ & $\begin{array}{l}2 \% 00 \mathrm{~g} \\
\text { gemischte } \\
\text { Nahrung }\end{array}$ & $\begin{array}{c}1 \text { Uhr } \\
1,1 \text { ccm Adren: }\end{array}$ & $\begin{array}{c}\text { bis 10. III. } \\
122 \text { ccm Harn } \\
\text { mit } 1,05 \mathrm{~g} \text { Zucker }\end{array}$ & & \\
\hline 10. III. & & $\mid \begin{array}{c}12 \text { Uhr } 35-42 \\
15 . \text { ccm } \\
25 \% \text { Na2SO } \\
1 \text { Uhr } 30-37 \\
13 \text { ecm } \\
25 \% \mathrm{Na}_{2} \mathrm{SO}_{4} \\
2 \text { Uhr } 40 \\
1 \mathrm{ccm} \text { Adrenalin }\end{array}$ & $\begin{array}{l}\text { von } 2 \text { Uhr } 40 \\
\text { bis } 5 \text { Uhr } 30 \\
55 \mathrm{cem} \text { Harn } \\
\text { mit } 0,1 \mathrm{~g} \text { Zucker }\end{array}$ & & \\
\hline $\begin{array}{l}\text { XXVII. } \\
\text { 14. III. }\end{array}$ & $\begin{array}{l}2700 \mathrm{~g} \\
\text { gemischte } \\
\text { Nahrung }\end{array}$ & $\begin{array}{l}4 \text { Uhr } 30 \\
1,4 \mathrm{ccm} \\
\text { Adrenalin }\end{array}$ & $\begin{array}{c}\text { bis } 15 . \text { III. } \\
210 \mathrm{cem} \text { Harn } \\
\text { mit } 6,6 \mathrm{~g} \text { Zucker }\end{array}$ & & \\
\hline 15. III. & & $\begin{array}{c}4 \mathrm{Uhr} 40-50 \\
14 \mathrm{~cm} \\
25 \% \mathrm{Na}_{2} \mathrm{SO}_{4} \\
5 \mathrm{Uhr} 50 \\
1,4 \mathrm{ccm} \\
\text { Adrenalin }\end{array}$ & $\begin{array}{c}\text { von } 15 . \text { III. } \\
5 \text { Uhr } 50 \\
\text { bis } 16 \text { III. } \\
220 \text { ccm zucker- } \\
\text { freier Harn }\end{array}$ & \begin{tabular}{|c|}
$8 \mathrm{Uhr}$ \\
Blutentnahme \\
$0,328 \%$ \\
Zucker
\end{tabular} & \\
\hline
\end{tabular}




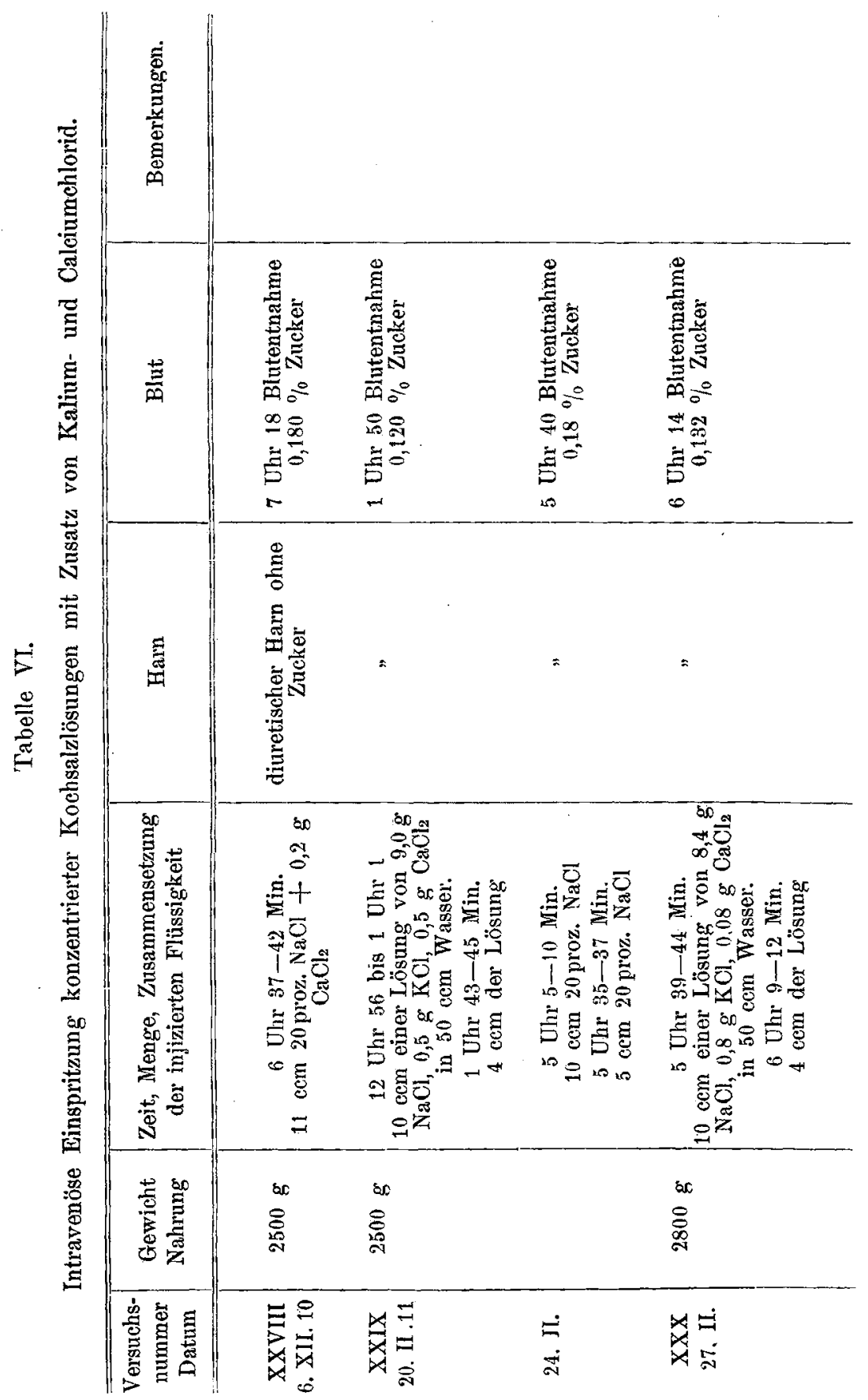


Zur Wirkung intrav. Einspritzungen von konz. Salz- und Zuckerlösungen. 153

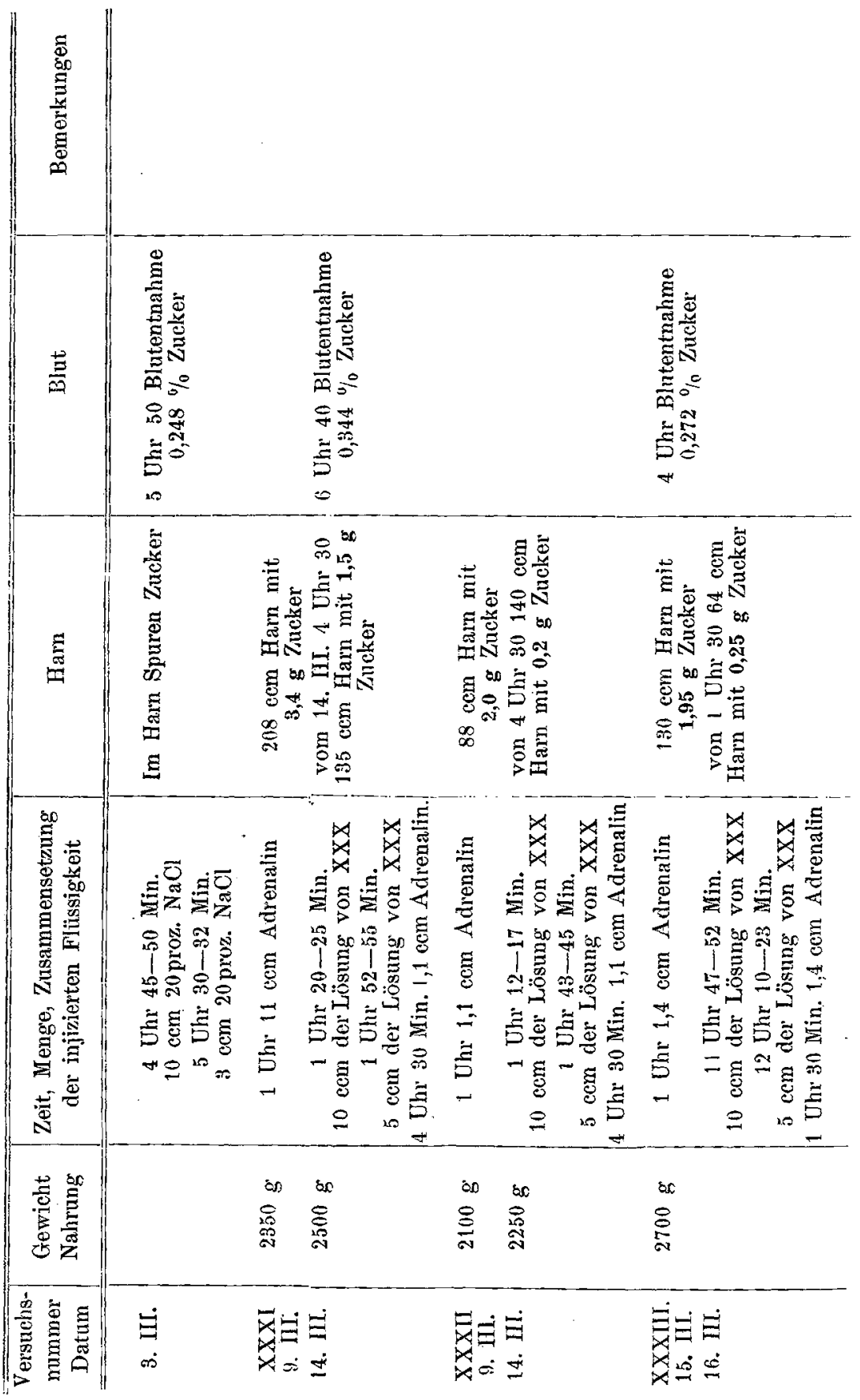


Bei näherer Analyse der eingangs erwähnten Beobachtung über den berabsetzenden Einfluß intravenös eingeführter Salzlösungen auf die Adrenalinglykosurie, hat sich herausgestellt, daß es sich hier um eine gesteigerte Undurchlässigkeit der Niere für den Blutzucker handelt. Um das konstante Auftreten dieser Erscheinung zu sichern, ist es vorteilhaft, die Salzlösungen in einstïndigen Intervallen zwei bis dreimal einzuführen und das Adrenalin erst später einzuspritzen. Näheres in Tabelle VII.

Wir sehen, daß die intravenöse Kochsalzeinfïhrung die Adrenalinhyperglykämie ungestört auftreten lälst; der Harnzucker ist aber sehr bedeutend herabgedrückt. Dieselben Kaninchen scheiden bei sonst gleichen Bedingungen viel größere Zuckermengen im Harne aus, wenn dieselbe Adrenalinmenge allein eingespritzt wurde. Wir sind berechtigt anzunehmen, daß die Nieren unter dem Einflusse der eingeführten Kochsalzlösung auch für den Zucker der Adrenalinhyperglykämie weniger durchgängig geworden sind. Es war jetzt möglich, die Funktion des Kations mit Bezug auf die Durchlässigkeit der Niere für Zucker einer Prüfung zu unterziehen. Wir haben der NaClLösung $\mathrm{KCl}$ und $\mathrm{CaCl}_{2}$ zugesetzt und diese intravenös eingefiihrt; nachber wurde genau wie bei den Versuchen mit reinem $\mathrm{NaCl}$ das Adrenalin eingespritzt und die Zuckermenge im Harne und Blute festgestellt.

Die Tahelle VI zeigt, daß die Durchlässigkeit der Niere für $\mathrm{Zu}$ cker bei Einführung von $\mathrm{NaCl}+\mathrm{KCl}+\mathrm{CaCl}_{2}$ dieselben Veränderungen erleidet wie bei reinem $\mathrm{NaCl}$ ind ist also a uch vom Kation unabängig.

Es ist anzunehmen, dab die beobachteten Veränderungen in der Funktion der Niere lediglich dureb die osmotischen Wirkungen der eingeführten Salzlösungen hervorgerufen werden. Der physikalische Zustand des Nierenzellenprotoplasmas wird wahrscheinlich durch Schwankungen seines Wassergehaltes derart verändert, daß die großen Zuckermoleküle es nicht passieren können. Die Veränderung kann dann wieder zurücktreten (Versuch 6).

Es ist zu beachten, dah dasselbe Agens gleichzeitig eine Hyperglykämie und eine Herabsetzung der Durchlässigkeit der Niere für Zucker hervorrufen kann; es sind dabei verschiedene Mechanismen tätig.

Angesichts dessen, daß für die Durchlässigkeit der Niere osmotische Faktoren maßgebend sind, lag der Gedanke nahe, zu prüfen, 
ob auch der osmotische Einfluh, der durch die Anwesenheit einer größeren Zuckermenge im Blute bedingt ist, sich iñ ähnlicher Weise geltend machen kann. Freilich mußte in Betracht gezogen werden, daß die intravenöse Einspritzung von Zuckerlösungen den Versuch erheblich kompliziert und einheitliche Resultate nicht zu erwarten sind. Es ist bekannt, dah bei intravenöser Einfuihrung konzentrierter Zuckerlösungen nur ein kleiner Teil die Niere passiert (Brassol (13) eigene Versuche Tier XXXVI), ein Teil des Zuckers wird als Glykogen in der Leber deponiert, (nach Popielski 14 bis 20 Proz). Über das Schicksal der restlichen Glykose sind wir nicht genau unterrichtet; im Blute jedenfalls befindet sie sich nicht. Die osmotischen Schwankungen im Blute können nur kurzdauernd sein und andererseits kann das große Glykogendepot der Leber und die dadurch bedingte starke Hyperglykämiefähigkeit der Versuchstiere kleine Durchlässigkeitsveränderungen in der Niere ganz verdecken.

Aus den zablreichen Versuchen, die in dieser Richtung ausgeführt wurden und die tatsächlich verschiedenartig ausgefallen sind, führen wir einige als Beispiel an. (Tab. VIII).

Es ist aus den angegebenen Zahlen jedenfalls zu entnehmen, daß die Anwesenheit größerer Zuckermengen im Blute die Durchlässigkeit der Niere für Glykose verändern kann. Dieser Vorgang äußert sich einmal als gesteigerte Durchlässigkeit (Tier 37), wo Glykosurie trotz normaler Blutzucker-Verhältnisse auftritt (dasselbe berichtet Brassol bei Hunden beobachtet zu haben); ein anderes Mal als verminderte Durchlässigkeit (Tier 34 und 35), wo trotz bedeutender Hyperglykämie die Adrenalinglykosurie herabgesetzt ist.

Da die Wirkung der Zuekerlösung auf den osmotischen Druck viel schwächer sein muß als diejenige der Salzlösungen, da weiter bei den Salzlösungen nur die verminderte Durchlässigkeit der Niere iür Zucker zum Vorschein kommt 1), ist anzunehmen, daß beide Veränderungen nur Stadien desselben im. Protoplasma der Nierenzellen vor sich gebenden Prozesses bilden, und es ist wabrscheinlich, daß die gesteigerte Durchlässigkeit als die erste Phase aufzufassen ist:

Die Bedeutung der angeführten Ergebnisse für die Pathologie des menschlichen Diabetes wird an anderer Stelle näher erörtert werden. werden.

1) Versuch X kann als solcher mit gesteigerter Durchlässigkeit gedeutet 


\section{Tabelle VII.}

Versuche über den Einfluß der intravenösen Einspritzung von konzentrierten Kochsalzlösungen auf die Adrenalinglykosurie. Die Kochsalzlösung war 20 prozentig. Sonst wie Tabelle I.

\begin{tabular}{|c|c|c|c|c|c|}
\hline $\begin{array}{c}\text { Versuchs- } \\
\text { nummer } \\
\text { Datum }\end{array}$ & $\begin{array}{l}\text { Gewicht } \\
\text { Nahrung }\end{array}$ & $\begin{array}{l}\text { Zeit, Menge der inji- } \\
\text { zierten Flüssigkeit. }\end{array}$ & Harn & Blut & 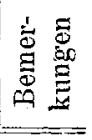 \\
\hline 26. XI. 09 & $\begin{array}{r}3320 \mathrm{~g} \\
\text { weiße } \\
\text { Rüben }\end{array}$ & 1,5 ccm Adrenalín & $\begin{array}{c}\text { enthält } 5,7 \mathrm{~g} \\
\text { Zucker }\end{array}$ & & \\
\hline 30. XI. & $\begin{array}{l}3320 \mathrm{~g} \\
\text { Rüben }\end{array}$ & $\begin{array}{c}10 \text { Uhr } 32-42 \\
10 \mathrm{ccm} 20 \text { proz. } \mathrm{NaCl} \\
11 \text { Uhr } 20 \\
1,5 \mathrm{ccm} \text { Adrenalin } \\
\text {, }\end{array}$ & $\begin{array}{c}\text { von 11 Uhr } 20 \\
\text { Harn gesammelt } \\
\text { enthält } 2,5 \mathrm{~g} \\
\text { Zucker }\end{array}$ & $\begin{array}{l}1 \text { Uhr } 20 \text { Blut- } \\
\text { entnahme } \\
0,453 \% \\
\text { Zucker }\end{array}$ & \\
\hline $\begin{array}{l}\text { Ii. } \\
\text { 7. } \mathrm{XH} .\end{array}$ & $\begin{array}{l}2700 \mathrm{~g} \\
\text { Rüben }\end{array}$ & $\begin{array}{c}11 \text { Uhr } \\
1,5 \mathrm{ccm} \text { Adrenalin }\end{array}$ & $\begin{array}{l}187 \text { ccm Harn } \\
2,4 \text { g Zucker }\end{array}$ & \begin{tabular}{|}
1 Uhr Blut- \\
entnahme \\
$0,323 \%$ \\
Zucker
\end{tabular} & \\
\hline 13. XII. & Rüben & $\begin{array}{l}3 \mathrm{Uhr} 3-6 \\
9 \mathrm{ccm} 20 \text { proz. } \mathrm{NaCl}\end{array}$ & & & \\
\hline 14. XII. & $2220 \mathrm{~g}$ & $\begin{array}{c}11 \text { Uhr } 15-21 \\
8 \mathrm{ecm} 20 \text { proz. NaCl } \\
12 \text { Uhr 5 } \\
1,4 \mathrm{cem} \text { Adrenalin }\end{array}$ & $\mid \begin{array}{c}\text { von } 11 \text { Uhr } 15 \\
163 \text { ccm Harn } \\
\text { mit 0,95 g Zucker }\end{array}$ & \begin{tabular}{|}
2 Uhr 5 Blut- \\
entnahme \\
$0,350 \%$ \\
Zucker
\end{tabular} & \\
\hline $\begin{array}{l}\text { III. } \\
\text { 7. I. } 1910\end{array}$ & $\begin{array}{l}3050 \mathrm{~g} \\
\text { Rüben }\end{array}$ & $\begin{array}{l}11 \text { Uhr } \\
1,5 \text { ccm Adrenalin }\end{array}$ & $\begin{array}{c}264 \mathrm{cem} \text { Harn } \\
\text { m. } 6,2 \mathrm{~g} \text { Zucker }\end{array}$ & $\begin{array}{l}1 \text { Uhr Blut- } \\
\text { entnahme } \\
\mathbf{0 , 4 9 5} \% \\
\text { Zucker }\end{array}$ & \\
\hline $\begin{array}{l}\text { 10. I. } \\
\text { 11. I. }\end{array}$ & & $\begin{array}{l}11 \text { Uhr } 10-19 \\
10 \text { ccm } 20 \text { proz. } \mathrm{NaCl} \\
12 \text { Uhr } 20-30 \\
10 \text { ccm } 20 \text { proz. } \mathrm{NaCl}\end{array}$ & & & \\
\hline 12. $\mathrm{I}$. & $2600 \mathrm{~g}$ & $\begin{array}{c}11-11 \text { Uhr } 10 \\
10 \mathrm{ccm} 20 \text { proz. NaCl } \\
1 \text { Uhr } \\
1,3 \mathrm{ccm} \text { Adrenalin }\end{array}$ & $\begin{array}{c}\text { von } 1 \text { Uhr } \\
195 \text { ccm Harn } \\
\text { mit } \mathbf{1 , 6 8} \mathrm{g} \\
\text { Zucker }\end{array}$ & \begin{tabular}{|c|}
3 Uhr Blut- \\
entnahme \\
$0,40 \%$ \\
Zncker
\end{tabular} & \\
\hline $\begin{array}{l}\text { IV. } \\
\text { 14. IV. }\end{array}$ & $\begin{array}{l}2200 \mathrm{~g} \\
\text { Kar- } \\
\text { toffeln }\end{array}$ & 1, ə̀ ccm Adrenalin & $\begin{array}{l}100 \mathrm{ccm} \text { Harn } \\
\text { mit } 6 \mathrm{~g} \text { Zucker }\end{array}$ & & \\
\hline
\end{tabular}


Zur Wirkung intrav. Einspritzungen von konz. Salz- und Zuckerlösungen. 157

\begin{tabular}{|c|c|c|c|c|c|}
\hline $\begin{array}{c}\text { Versuchs- } \\
\text { nummer } \\
\text { Datum }\end{array}$ & $\begin{array}{l}\text { Gewicht } \\
\text { Nahrung }\end{array}$ & $\begin{array}{l}\text { Zeit, Menge der inji- } \\
\text { zierten Flüssigkeit. }\end{array}$ & Harn & Blut & 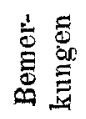 \\
\hline 16. IV. & & $\begin{array}{l}11 \text { Uhr } 15-23 \\
10 \mathrm{ccm} 20 \text { proz. NaCl } \\
12 \text { Uhr } 20-28 \\
10 \mathrm{ccm} 20 \text { proz. NaCl } \\
1 \text { Uhr } \\
1,5 \mathrm{ccm} \text { Adrenalin }\end{array}$ & $\begin{array}{c}\text { von } 1 \text { Thr } \\
125 \text { cem Harn } \\
\text { mit } 1,25 \mathrm{~g} \\
\text { Zucker }\end{array}$ & & \\
\hline $\begin{array}{l}\text { V } \\
\text { 5. XII. }\end{array}$ & $\begin{array}{l}2200 \mathrm{~g} \\
\text { Weißkr. }\end{array}$ & 1,1 cem Adrenalin & $\begin{array}{c}15 \bar{i} \text { ccm Harn } \\
\text { mit } 2,7 \mathrm{~g} \text { Zucker }\end{array}$ & & \\
\hline 7. XII. & $2150 \mathrm{~g}$ & $\left\{\begin{array}{c}11 \text { Uhr } 35-45 \\
10 \mathrm{ccm} 20 \text { proz. NaCl } \\
1 \text { Uhr } 4-9 \\
10 \mathrm{ccm} 20 \text { proz. NaCl } \\
2 \text { Uhr } 45 \\
1,1 \mathrm{ccm} \text { Adrenalin }\end{array}\right.$ & $\begin{array}{l}\text { von } 2 \text { Uhr } 45 \\
31 \text { ecm Harn } \\
\text { ohne Zucker }\end{array}$ & & \\
\hline $\begin{array}{l}\text { VI. } \\
\text { 5. XII. }\end{array}$ & $\begin{array}{c}1950 \mathrm{~g} \\
\text { Weißkr. }\end{array}$ & 1 ccm Adrenalin & $\begin{array}{c}106 \mathrm{cem} \text { Harn m. } \\
\mathbf{2 , 8} \mathrm{g} \text { Zucker }\end{array}$ & & \\
\hline т. XII. & $1900 \mathrm{~g}$ & $\mid \begin{array}{c}11 \text { Uhr } 25-30 \\
11 \mathrm{ccm} 20 \text { proz. NaCl} \\
12 \text { Uhr } 45-50 \\
11 \mathrm{ccm} 20 \text { proz. } \mathrm{NaCl} \\
2 \text { Uhr } 45 \\
1 \mathrm{ccm} \text { Adrenalin }\end{array}$ & $\begin{array}{c}\text { von } 2 \text { Uhr } 45 \\
58 \mathrm{ccm} \text { Harn } \\
\text { mit } 0,46 \mathrm{~g} \\
\text { Zucker }\end{array}$ & & \\
\hline 10. XH. & & $1 \mathrm{ccm}$ Adrenalin & $\begin{array}{c}280 \mathrm{ccm} \text { Harn m. } \\
3,4 \mathrm{~g} \text { Zucker }\end{array}$ & & \\
\hline 12. XH. & $1920 \mathrm{~g}$ & $\begin{array}{l}12 \text { Uhr } 15-18 \\
11 \text { ccm } 20 \text { proz. NaCl } \\
1 \text { Uhr } 15-17 \\
5 \text { ccm } 20 \text { proz. NaCl } \\
2 \text { Uhr } 15-17 \\
5 \mathrm{ccm} 20 \text { proz. NaCl } \\
2 \text { Uhr } 37 \\
1 \text { ccm Adrenalin }\end{array}$ & $\begin{array}{c}\text { von } 2 \text { Uhr } 37 \\
\text { bis } 5 \text { Uhr } 35 \mathrm{im} \\
\text { Harn kein } \\
\text { Zucker }\end{array}$ & $\begin{array}{c}4 \text { Uhr 30 } \\
\text { Blutentnabm. } \\
\mathbf{0 , 3 1 6} \% \\
\text { Zucker }\end{array}$ & \\
\hline $\begin{array}{l}\text { XVIa. } \\
\text { 5. XII. }\end{array}$ & $\begin{array}{l}2400 \mathrm{~g} \\
\text { Weißkx. }\end{array}$ & $1,2 \mathrm{ccm}$ Adrenalin & $294 \mathrm{ccm}$ Harn m. & & \\
\hline 13. X11. & & $\begin{array}{l}11 \text { Uhr } 11-15 \\
11 \text { ccm } 20 \text { proz. } \mathrm{NaCl} \\
12 \text { Uhr } 15-18 \\
9 \mathrm{ccm} 20 \text { proz. } \mathrm{NaCl} \\
12 \text { Uhr } 45-50 \\
5 \mathrm{ccm} 20 \text { proz. } \mathrm{NaCl} \\
3 \text { Uhr } \\
1,2 \mathrm{ccm} \text { Adrenalin }\end{array}$ & $\begin{array}{c}\text { von 3 Uhr } 85 \mathrm{ccm} \\
\text { Harn ohne } \\
\text { Zucker }\end{array}$ & $\begin{array}{c}5 \text { Uhr 15 } \\
\text { Blutentnabm. } \\
\mathbf{0 , 2 0 8} \% \\
\text { Zucker }\end{array}$ & \\
\hline
\end{tabular}


Tabelle VIII.

Intravenöse Einführung konzentrierter Glykoselösungen (40\%).

\begin{tabular}{|c|c|c|c|c|c|}
\hline $\begin{array}{l}\text { Versuchs- } \\
\text { numnacr } \\
\text { Datum }\end{array}$ & Gewicht & $\begin{array}{c}\text { Zeit, Menge der in- } \\
\text { jizierten Flüssigkeiten. }\end{array}$ & Hara & Blut & 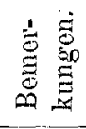 \\
\hline $\begin{array}{l}\text { XXXIV } \\
20 . \text { II. } 11\end{array}$ & $2800 \mathrm{~g}$ & $\begin{array}{c}\text { 4,30 Uhr } 30 \text { 1,5 ccm } \\
\text { Adrenalin }\end{array}$ & $\begin{array}{c}\text { bis } 21 \text {. II } 270 \mathrm{ccm} \\
\text { Harn mit 3,34 g } \\
\text { Zucker }\end{array}$ & & \\
\hline 21. II. & $2800 \mathrm{~g}$ & $\begin{array}{c}1 \text { Uhr } 7-12 \\
14 \text { ccm Glykoselösung } \\
1 \text { Uhr } 45-51 \\
14 \text { ccm Glykoselösung } \\
2 \text { Uhr 19-21 } \\
7 \text { ccm Glykoselösung } \\
3 \text { Uhr } \\
1,5 \text { ccm Adrenalin }\end{array}$ & $\begin{array}{c}\text { von } 3 \text { Uhr bis } \\
22 \text {.I. } 151 \mathrm{ecm} \\
\text { Harn mit } \mathbf{1 , 6 2} \mathbf{g} \\
\text { Zucker }\end{array}$ & $\begin{array}{c}5 \text { Uhr } 55 \\
\text { Blutentnahm. } \\
0,342 \% \\
\text { Zucker }\end{array}$ & \\
\hline $\begin{array}{l}\text { XXXV } \\
27.11 .\end{array}$ & $2550 \mathrm{~g}$ & $\begin{array}{c}6 \text { Uhr } 30 \\
1,1 \mathrm{cem} \text { Adrenalin }\end{array}$ & $\left|\begin{array}{c}\text { bis } 28 . \text { II. } 103 \mathrm{ccm} \\
\text { Harn mit } 4,02 \mathrm{~g} \\
\text { Zucker }\end{array}\right|$ & & \\
\hline 28. II. & & \begin{tabular}{|}
12 Uhr $15-19$ \\
10,5 ccm Glykoselösung \\
12 Uhr $45-50$ \\
11 cem Glykoselösung \\
1 Uhr $24-27$ \\
5,5 cem Glykoselösung \\
4 Uhr 10 \\
1,1 cem Adrenalin
\end{tabular} & $\begin{array}{l}\text { Harn v. } 4 \text { Uhr } 10 \\
\text { bis } 1.1 \mathrm{II} .76 \mathrm{ccm} \\
\text { mit } 1,52 \mathrm{~g} \text { Zuck. }\end{array}$ & \begin{tabular}{|c|}
6 Uhr 25 \\
Blutentnahm. \\
$0,442 \%$ \\
Zucker
\end{tabular} & \\
\hline $\begin{array}{c}\text { XXXVII } \\
28 . \mathrm{II}\end{array}$ & $2400 \mathrm{~g}$ & 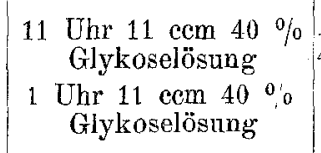 & $\begin{array}{l}\text { Harn von } 5 \text { Uhr } \\
45 \text { bis } 6 \text { Uhr } 40 \\
\text { zuckerhaltig }\end{array}$ & \begin{tabular}{|}
5 Uhr 45 \\
Blutentnahm. \\
$0,06 \%$ \\
Zucker
\end{tabular} & \\
\hline $\begin{array}{l}\text { XXXVIII } \\
28 . \text { II. }\end{array}$ & $2550 \mathrm{~g}$ & $\begin{array}{c}12 \text { Uhr } 15 \\
11 \text { ccm Glykoselösung } \\
12 \text { Uhr } 45 \\
11 \text { cem Glykoselösung } \\
1 \text { Uhr } 29 \\
5,5 \text { ccm Glykoselösung }\end{array}$ & $\begin{array}{l}\text { Harn bis } 4 \text { Uhr } \\
10 \text { enthält in } \\
83 \mathrm{cem} 1,4 \mathrm{~g} \\
\text { Zucker. folgend. } \\
\text { Harn zuckerfrei. }\end{array}$ & & \\
\hline
\end{tabular}


Zur Wirkung intrav. Einspritzungen von konz. Salz- unà Zuckerlösungen. 15̄9

Zusammenfassung.

1. Intravenöse Einführung konzentrierter Salzlösungen ruft durch Reizung des zentralen Nervensystems eine Hyperglykämie hervor, wobei die Muskeln, vielleicht auch die Leber, ibr Glykogen einbüßen.

2. Der Nervenreiz ist eine Kationwirkung.

3. Intravenöse Einführung konzentrierter Salzlösungen verändert die Durchlässigkeit der Niere für Zucker. Die Erscheinung ist durch osmotische Faktoren bedingt und verläuft, wahrscheinlich, in zwei Phasen: erst eine gesteigerte und dann eine verminderte Durchlässigkeit der Niere für Zucker.

4. Intravenöse Einfübrung konzentrierter Glykoselösungen ruft dieselbe, nur etwas schwächere, Veränderung der Nierendurchlässigkeit für Zucker hervor, wie die Salzlösungen.

\section{Literaturverzeichnis.}

1. Magnus, Archiv für experim. Pathol. u. Pharmak., Bd. 44 u. 61.

2. von den Velden, Zeitschr. für exper. Pathol. und Therapie, 1909.

3. Münzer, Archiv für experim. Pathol. und Pharmak., Bd. 41.

4. Wilen ko, Internat. Beiträge zur Pathol. und Therapie der Ernährungsstörungeu, Bd. 2, H. 2.

5. Tomaszewski u. Wilenko, Berlin, kl. Wochenschr. 1908, No. 26.

6. Pollak, Archiv für experim. Pathol. und Pharmak., Bd. 61, S. 149.

7. Schultze, Archiv für experim. Pathol. und Pharmak., Bd. 43.

8. Münzer l. c.

9. Physikalische Chemie und Nedizin von Richter u. Korangi, 1907.

10. Bock u. Hoffmann, Du Bois-Reymonds Ar., 1871.

11. Brow, Amer. Journ. of Physiol., 1904.

12. Fischer, Pflügers Archiv, Bd. 109.

13. Brassol, Archiv für Anat. und Physiol.; 1894, Physiol. Abteilung.

14. Popielski, Bolnitznaja gaseta Botkina, 1897. 